\title{
LEFT PERFECT RINGS THAT ARE RIGHT PERFECT AND A CHARACTERIZATION OF STEINITZ RINGS
}

\author{
WILLIAM H. RANT ${ }^{1}$
}

\begin{abstract}
A proof is given to show all flat left modules of a ring are free if and only if the ring is a local ring with a left $T$ nilpotent maximal ideal. We characterize left perfect rings whose radical $R$ has the property that $I R^{n}=\{0\}$ for some positive integer $n$ if $I$ is a finitely generated right ideal contained in $R$. We cite an example of a left perfect ring which does not have this property. It is shown that if the set of irreducible elements of a left perfect ring is right $T$-nilpotent then the ring is right perfect.
\end{abstract}

Introduction. If $R$ is the radical of a left perfect ring, an element of $R$ is called irreducible if it cannot be expressed as a product of two elements of $R$. If $R$ is the radical of a ring that is left and right perfect, we show each element of $R$ can be expressed as a product of irreducible elements. It follows that a perfect ring having a finite set of irreducible elements is right and left artinian and has a finite radical; furthermore if the ring is a local ring with a nonzero radical, the ring is finite. Throughout this paper we will assume that a ring has an identity element and that a module is unitary.

Definition. Let $F$ be a free left $A$-module having $\left\{u_{j}\right\}_{j=1}^{\infty}$ as a basis; let $\left\{a_{n}\right\}_{n=1}^{\infty}$ be a sequence in $A$ and let $G$ denote the submodule of $F$ generated by $\left\{u_{j}-a_{j} u_{j+1}\right\}_{j=1}^{\infty}$. The pair $F$ and $G$ will be denoted $\left[F,\left\{a_{n}\right\}, G\right]$.

Lemma 1. F/G is a flat left A-module.

Proof. It is sufficient to show that if $I$ is a right ideal, $I F \cap G=I G$. If $m \in I F \cap G$, set $m=\sum_{i=1}^{n} x_{i} u_{i}$ with $x_{i} \in I$, and $m=\sum_{i} b_{i}\left(u_{i}-a_{i} u_{i+1}\right)$ with $b_{i} \in A$. Expanding and comparing coefficients, $b_{1}=x_{1}, b_{2}=x_{2}+b_{1} a_{1}, \cdots$, $b_{n}=x_{n}+b_{n-1} a_{n-1}$, so $b_{i} \in I$ for $i=1,2, \cdots, n$ and $I F \cap G=I G$.

Notation. Let $R_{1}=\{x \in A: x$ does not have a right inverse $\}$ and $R_{2}=$ $\{x \in A: x$ does not have a left inverse $\}$.

Received by the editors February 16, 1971 and, in revised form, May 7, 1971.

AMS 1970 subject classifications. Primary 16A10, 16A48, 16A50; Secondary 16A46.

Key words and phrases. Perfect ring, Steinitz ring, T-nilpotent, local ring, radical, irreducible element.

${ }^{1}$ This paper consists primarily of portions of the author's dissertation, written at the University of Alabama under the direction of Dr. Joseph Neggers.

The author wishes to express appreciation for various helpful comments and observations made by the referee.

(c) American Mathematical Society 1972 
LEMMA 2. If each flat left A-module is projective, each nonunit is a right zero divisor. Moreover, $R_{1}=R_{2}$.

Proof. Let $0 \neq x \in R_{1}$ and let $\left\{a_{n}\right\}_{n=1}^{\infty}$ be the constant sequence with $a_{n}=x$. Consider $\left[F,\left\{a_{n}\right\}, G\right]$. Since $F / G$ is flat, it is projective and $G$ is a direct summand of $F$. It follows from [1] that the sequence of principal ideals $\left\{x^{n} A\right\}_{n=1}^{\infty}$ terminates, so $x^{n}=x^{n+1} y$ for some $y \in A$. Let $n$ be the smallest positive integer such that $x^{n}(1-x y)=0$. Since $x \in R_{1}, 1-x y \neq 0$. If $n=1$, we are through. If $n>1, x\left(x^{n-1}(1-x y)\right)=0$ and $x^{n-1}(1-x y) \neq 0$. Since each element of $R_{1}$ is a right zero divisor, $R_{1}=R_{2}$. The following lemma is a verification of a well-known fact established in [1].

LEMMA 3. If $M$ is a flat left module of a left perfect ring, $M$ is projective.

Proof. From [1], $M$ has a projective cover $f: P \rightarrow M$ with $K=\operatorname{ker}(f) \subseteq$ $R P$, where $R$ is the radical of $A$. Since $M$ is flat, $K \cap R P=R K$. This gives $K=R K$, so $K=\{0\}$ and $M$ is projective.

THEOREM 1. A is a local ring with a left T-nilpotent maximal ideal if and only if each flat left A-module is free.

Proof. If $A$ is a local ring whose maximal ideal is left $T$-nilpotent, $A$ is a left perfect ring. From Lemma 3 , each flat left module is projective and hence free.

For the converse let $\left\{a_{n}\right\}_{n=1}^{\infty}$ be a sequence of nonunits of $A$ and consider $\left[F,\left\{a_{n}\right\}, G\right]$. For $x \in F$ denote by $\bar{x}$ the corresponding element of $F / G$. Then $\left\{\bar{u}_{k}\right\}_{k=1}^{\infty}$ is a system of generators for $H=F / G$ with $\bar{u}_{k}=a_{k} \bar{u}_{k+1}$. Therefore any $b \in H$ is of the form $b=a \bar{u}_{n}$ for some $n$ with $a \in A$. It follows that $b=a a_{n} \bar{u}_{n+1} \in A a_{n} H$. Now $H$ is free, and suppose $b$ is an element of a basis $B$ of $H$. Then $b \in A a_{n} H$ gives $1 \in A a_{n} A$. This is impossible since $a_{n}$ is a nonunit and $R_{1}=R_{2}$ (Lemma 2). Thus $B$ has no elements and $H=\{0\}$. Therefore $F=G$, and expanding the relation $u_{1}=\sum_{k=1}^{n} b_{k}\left(u_{k}-a_{k} u_{k+1}\right)$ gives $a_{1} \cdots a_{n}=$ 0 . In particular any nonunit is nilpotent, so $A$ is a local ring with left $T$-nilpotent maximal ideal $R_{1}=R_{2}$ [3, Lemma 2].

Definition. Let $A$ be a left perfect ring with radical $R$ and let $x \in A$. Set $h(x)=0$ if $x R=\{0\}$, otherwise set

$$
h(x)=\sup \left\{n: x x_{1} \cdots x_{n} \neq 0 \text { for some } x_{i} \in R\right\} .
$$

LEMMA 4. Let $A$ be a left perfect ring. If $x, y \in A, h(x y) \leqq h(x)$ and $h(x+y) \leqq h(x)+h(y)$.

Proof. Clearly $h(x y) \leqq h(x)$. If $h(x+y) \neq 0,(x+y) x_{1} x_{2} \cdots x_{n} \neq 0$ for some $x_{i} \in R$, so $h(x) \geqq n$ or $h(y) \geqq n$, and

$$
h(x+y) \leqq \max \{h(x), h(y)\} \leqq h(x)+h(y) .
$$


THEOREM 2. If $A$ is a left perfect ring with radical $R, h(x)<\infty$ for each $x \in R$ if and only if for each finitely generated right ideal $I$ in $R$, $I R^{n}=\{0\}$ for some positive integer $n$.

Proof. Let $I=x_{1} A+\cdots+x_{n} A$ be a right ideal in $R$. If $y=x_{1} a_{1}+\cdots+$ $x_{n} a_{n} \in I, h(y) \leqq \max \left\{h\left(x_{i} a_{i}\right)\right\}_{i=1}^{n} \leqq \max \left\{h\left(x_{i}\right)\right\}_{i=1}^{n}$. If we set $K=\max \left\{h\left(x_{i}\right)\right\}_{i=1}^{n}$, $y R^{K+1}=\{0\}$. For the converse let $x \in R$ and suppose $h(x) \geqq 1$. Since $(x A) R^{n}=\{0\}$ for some $n, h(x)<\infty$.

In [1] Bass asked if $I R^{n}=\{0\}$ for some $n$, if $I$ is a finitely generated right ideal in $R$, the radical of a left perfect ring. From [2, p. 117] there exists a left perfect ring $A$ with an element $x$ in the radical $R$ such that $x A$ is not nilpotent. Clearly $(x A) R^{n} \supseteq(x A)^{n} \neq\{0\}$ for all $n$.

THEOREM 3. Let $A$ be a left and right perfect ring with radical $R$, each element of $R$ can be expressed as a product of irreducible elements.

Proof. Let $0 \neq x \in R$. If $x$ is irreducible, we are through; otherwise $x=x_{1} y_{1}$ for some $x_{1}, y_{1} \in R$. If $y_{1}$ is not irreducible we have $y_{1}=x_{2} y_{2}$ for some $x_{2}, y_{2} \in R$. Due to the left $T$-nilpotence of $R$ we have $x=x_{1} \cdots x_{n} y_{n}$ for some $n$, with $y_{n}$ irreducible; so $x$ can be expressed in the form $r_{1} p_{1}$ with $p_{1}$ irreducible. If $r_{1}$ is not irreducible, $r_{1}=r_{2} p_{2}$ with $p_{2}$ irreducible. Continuing this process we eventually have $x=r_{n} p_{n} \cdots p_{1}$ with the elements $p_{i}$ and $r_{n}$ irreducible.

THEOREM 4. Let $A$ be a left perfect ring with radical $R . A$ is right perfect if $R^{\prime}=\{r \in R: r$ is irreducible $\}$ is right T-nilpotent.

Proof. Let $\left\{a_{i}\right\}_{i=1}^{\infty}$ be a sequence in $R$. By induction we show any nonzero product $a_{n} a_{n-1} \cdots a_{1}$ can be expressed in the form $a_{n}^{\prime} p_{n} \cdots p_{1}$, with $a_{n}^{\prime} \in A$ and each $p_{i}$ irreducible. If $n=1$, we refer to Theorem 3. Assume $a_{n} a_{n-1} \cdots a_{1}=a_{n}^{\prime} p_{n} \cdots p_{1}$ with each $p_{i}$ irreducible and suppose $a_{n+1} a_{n} \cdots$ $a_{1} \neq 0$. Since $a_{n+1} a_{n}^{\prime} \in R, a_{n+1} a_{n}^{\prime}=a p_{n+1}$ for some irreducible element $p_{n+1}$. Due to the right $T$-nilpotence of $\left\{p_{i}\right\}_{i=1}^{\infty}$ we have $a_{n} \cdots a_{1}=0$ for some $n$.

Lemma 11 in [5] shows that if $A$ is a left perfect ring with radical $R$ and $R / R^{2}$ is finitely generated as a left (right) $A$-module, then $A$ is left (right) artinian. Thus we have

THEOREM 5. If $A$ is a left perfect ring whose radical $R$ has only a finite set of irreducible elements, then $A$ is left and right artinian and $R$ is finite. Moreover, if $A$ is a local ring and $R \neq\{0\}, A$ is finite.

Proof. Since the set of irreducible elements $R^{\prime}$ is finite, clearly $R / R^{2}$ is finite, so $A$ is left and right artinian and $R^{N}=\{0\}$ for some $N$. From Theorem 3, each nonzero element of $R$ can be expressed as a product of at most $N-1$ elements from $R^{\prime}$, so $R$ is finite. It is easily seen that a local 
ring having a finite set of nonunits (but containing at least two elements) is finite.

Definition. From [3], a ring is a left Steinitz ring if each linearly independent subset of a free left module can be extended to a basis by adjoining elements of a given basis.

It is known that $A$ is a left Steinitz ring if and only if $A$ is a local ring with a left $T$-nilpotent maximal ideal. Hence $A$ is a left Steinitz ring if and only if $A$ is a left perfect local ring. A commutative perfect ring is a direct sum of a finite number of Steinitz rings [1]. It follows that a commutative perfect ring is artinian if its maximal ideals are finitely generated.

\section{REFERENCES}

1. H. Bass, Finitistic dimension and a homological generalization of semi-primary rings, Trans. Amer. Math. Soc. 95 (1960), 466-488. MR 28 \#1212.

2. J.-E. Björk, Rings satisfying a minimum condition on principal ideals, J. Reine Angew. Math. 236 (1969), 112-119. MR 40 \#1419.

3. B.-S. Chwe and J. Neggers, On the extension of linearly independent subsets of free modules to bases, Proc. Amer. Math. Soc. 24 (1970), 466-470. MR 40 \#5652.

4. V. E. Govorov, Rings over which flat modules are free, Dokl. Akad. Nauk SSSR 144 (1962), 965-967=Soviet Math. Dokl. 3 (1962), 836-838. MR 25 \#3076.

5. B. L. Osofsky, A generalization of quasi-Frobenius rings, J. Algebra 4 (1966), 373-387. MR 34 \#4305.

University of Alabama, University, Alabama 35486

Jacksonville State University, Jacksonville, Alabama 36265 